\title{
FINITE RANK RIESZ OPERATORS
}

\author{
U. KOUMBA and H. RAUBENHEIMER \\ Department of Mathematics, University of Johannesburg, APK Campus, Aucklandpark 2006, South Africa \\ e-mails:uakoumba@uj.ac.za; heinrichr@uj.ac.za
}

(Received 12 July 2012; accepted 24 January 2013; first published online 2 September 2013)

\begin{abstract}
We provide conditions under which a Riesz operator defined on a Banach space is a finite rank operator.
\end{abstract}

2010 Mathematics Subject Classification. 47B06, 46L05.

1. Introduction. Let $X$ be a Banach space, and denote by $\mathcal{L}(X)$ the Banach algebra of all bounded linear operators on $X$. An operator $T \in \mathcal{L}(X)$ is called a Riesz operator if the coset $T+\mathcal{K}(X)$ is quasinilpotent in the quotient algebra $\mathcal{L}(X) / \mathcal{K}(X)$, where $\mathcal{K}(X)$ is the closed ideal of compact operators in $\mathcal{L}(X)$. We refer the reader to Dowson [[4], Part 2] for some basic properties of Riesz operators. For $T \in \mathcal{L}(X)$, denote the null space of $T$ by $N(T)$ and the range of $T$ by $R(T)$. The smallest integer $n$ such that $N\left(T^{n}\right)=N\left(T^{n+1}\right)$ is called the ascent of $T$ and it is denoted by $\alpha(T)$. The descent of $T$ is the smallest integer $n$ such that $R\left(T^{n}\right)=R\left(T^{n+1}\right)$ and it is denoted by $\delta(T)$. If $M$ is a closed subspace of $X$ invariant under $T$ (i.e. $T(M) \subseteq M$ ), then the operator $T_{M}$ defined in $\mathcal{L}(X / M)$ by $T_{M}(x+M)=T x+M$ is called the induced operator of $T$ by $M$. The restriction of $T$ to $M$ is denoted by $\left.T\right|_{M}$. If $A$ is a $\mathrm{C}^{*}$-algebra then the operator $T \in \mathcal{L}(A)$ is said to be a homomorphism whenever $T(x y)=T x T y$ for all $x, y \in A$.

2. Riesz operators. It is well known that a compact operator on a Banach space is a finite rank operator if it has a closed range [1, Theorem 2.2.5]. We illustrate with an example that this is not necessarily true for Riesz operators.

EXAmPLE 2.1. Let $X$ be an infinite dimensional Banach space and $Y=X \times X$. Define an operator $T: Y \rightarrow Y$ by $T\left(x_{1}, x_{2}\right)=\left(x_{2}, 0\right)$ for all $\left(x_{1}, x_{2}\right) \in Y$. Then $T$ is a Riesz operator which is not of finite rank.

Since $T^{2}=0$, it follows from the definition of a Riesz operator that $T$ is a Riesz operator. Note that $T$ has a closed range, but $T$ is not a finite rank operator.

We are now going to provide conditions under which a Riesz operator is a finite rank operator.

THEOREM 2.2. Let $X$ be a Banach space and $T \in \mathcal{L}(X)$ a Riesz operator with $\alpha(T)=p$. If $R\left(T^{p}\right)+N\left(T^{p}\right)$ is closed in $X$, then $T^{p}$ is a finite rank operator.

Proof. If $N=N\left(T^{p}\right)$ then $\tilde{X}=X / N$ is a Banach space. Note that the operator $\widetilde{T}: \widetilde{X} \rightarrow X$ defined by $\widetilde{T}(x+N)=T^{p} x(x \in X)$ is well defined and that $T^{p}=\widetilde{T} \circ \pi$, where $\pi: X \rightarrow \widetilde{X}$ is the canonical quotient map. Since $N$ is a closed invariant subspace of $X$ under $T^{p},\left(T^{p}\right)_{N}$ defined on $\widetilde{X}$ by $T_{N}^{p}(x+N)=T^{p} x+N$ is a well-defined operator too. Also, note that $\left(T^{p}\right)_{N}$ is Riesz operator [4, Theorem 3.23]. It is not difficult to see 
that $\left(T^{p}\right)_{N}$ is one-to-one and deduce that its inverse $\left(T^{-p}\right)_{N}$ exists as a linear operator defined on $R\left(\left(T^{p}\right)_{N}\right)$. Since $R\left(T^{p}\right)+N\left(T^{p}\right)$ is closed in $X$, it follows from [8, Theorem II.5.2] that $\pi\left(R\left(T^{p}\right)\right)$ is closed in $\widetilde{X}$. This together with $R\left(\left(T^{p}\right)_{N}\right)=\pi\left(R\left(T^{p}\right)\right)$ gives that $\left(T^{p}\right)_{N}$ has a closed range. Therefore, the identity $I_{N}=T_{N}^{-p} \circ T_{N}^{p}$ is also a Riesz operator on $\widetilde{X}$ and this implies that $\widetilde{X}$ is finite dimensional. Since $R\left(T^{p}\right)=R(\widetilde{T})$ is finite dimensional, we are done.

Corollary 2.3. Let $T$ be a Riesz operator on a Banach space $X$ with $\alpha(T)=\delta(T)=$ p. Then $T^{p}$ is a finite rank operator.

Proof. Note that $X=R\left(T^{p}\right) \oplus N\left(T^{p}\right)$ whenever $\alpha(T)=\delta(T)=p$ [4, Proposition 1.51]. Since $R\left(T^{p}\right) \oplus N\left(T^{p}\right)$ is closed in $X$, it follows from Theorem 2.2 that $T^{p}$ is a finite rank operator.

One can provide an alternative proof for the above corollary by using the restriction operator instead of the induced operator. Indeed, if $\alpha(T)=\delta(T)=p$ then one can again decompose $X$ as a direct $\operatorname{sum} X=M \oplus N$, where $M=R\left(T^{p}\right)$ and $N=N\left(T^{p}\right)$. In view of [2, Lemma 3.4.2], $M$ and $N$ are closed invariant subspaces under $T$. Hence, $\left.T\right|_{M}$ and $\left.T\right|_{N}$ are Riesz operators [4, Theorem 3.21]. Define projections $P_{M}: X \rightarrow M$ and $P_{N}: X \rightarrow N$ by $P_{M}\left(x_{1}+x_{2}\right)=x_{1}$ and $P_{N}\left(x_{1}+x_{2}\right)=x_{2}$, for all $x_{1}+x_{2} \in M \oplus N$. If $T^{M}=\left(\left.T\right|_{M}\right) P_{M}$ and $T^{N}=\left(\left.T\right|_{N}\right) P_{N}$, then $T=T^{M}+T^{N}$ and $T^{p}=\left(T^{M}\right)^{p}$ since $T^{N}$ is nilpotent and $T^{M} T^{N}=T^{N} T^{M}=0$. Hence, by [2, Lemma 3.4.2] $T^{p}$ is a bijective Riesz operator and consequently $T^{p}$ is a finite rank operator.

3. $C^{*}$-algebras. Ghahramani in $[6$, Theorem 1] proved that a compact homomorphisms on a $C^{*}$-algebra is a finite rank operator. Mathieu in [7] generalized this result by proving that a weakly compact homomorphism defined on a $C^{*}$-algebra with range in a normed algebra is a finite rank operator. For an analytical proof of this fact, we refer the reader to Galé and Ransford in [5, Theorem 3.1]. In this section we are going to investigate to what extent the result of Ghahramani [6] can be generalized to Riesz operators on $C^{*}$-algebras that are also homomorphisms. Note that, in general, Riesz operators need not be weakly compact and weakly compact operators need not be Riesz operators. Firstly, we illustrate with an example that a Riesz operator defined on a $C^{*}$-algebra that is also a homomorphism need not be a finite rank operator.

EXAMPLE 3.1. Let $A$ be a non-unital $C^{*}$-algebra and $B$ be the $C^{*}$-algebra defined by $B=A \times A$. The operator $S: B \rightarrow B$ defined by $S(x, y)=(y, 0)$ for all $(x, y) \in B$ is a Riesz operator and also a homomorphism. But $S$ is not a finite rank operator.

By Example 2.1, $S$ is a Riesz operator that is not a finite rank operator. However, it is easy to verify that $S$ is also a homomorphism. By adjoining an identity to $B$ in the above example, one can construct an unital $C^{*}$-algebra denoted by $\mathbb{C} \mathbf{1} \oplus B$ and prove that $S$ can be extended to a Riesz operator $\bar{S}$ defined by $\bar{S}(\lambda,(x, y))=(\lambda, S(x, y))$ for all $(\lambda, x) \in \mathbb{C} 1 \oplus B$. Again, it is straightforward to show that $\bar{S}$ is a homomorphism. To prove that $\bar{S}$ is a Riesz operator, it suffices to define a projection $P$ on $\mathbb{C} 1 \oplus B$ by $P(\lambda, x)=(\lambda, 0)$ for all $(\lambda, x) \in \mathbb{C} \mathbf{1} \oplus B$ and to consider two operators $S_{1}$ and $S_{2}$ defined by $S_{1}=\bar{S} P$ and $S_{2}=\bar{S}(I-P)$, respectively. Note that $\bar{S}$ and $P$ commute and $\bar{S}=S_{1}+S_{2}$. Also, note that $S_{1}$ is a rank one operator and $S_{2}^{2}=0$. Then using the 
definition of a Riesz operator, it follows that $\bar{S}$ is a Riesz operator but $\bar{S}$ is not a finite rank operator.

In our next results, we are going to provide conditions under which a homomorphism that is also a Riesz operator defined on a $C^{*}$-algebra is a finite rank operator.

Proposition 3.2. Let $A$ be a $C^{*}$-algebra and $T: A \rightarrow A$ a Riesz operator. If $T$ is a monomorphism then $T$ is a finite rank operator.

Proof. It follows from [3, Theorem 5.4] that $T$ has a closed range. Hence, its inverse $T^{-1}$ is bounded and the identity $I=T^{-1} \circ T: A \rightarrow A$ is a Riesz operator too. So $A$ is finite dimensional and consequently $T$ is a finite rank operator.

Theorem 3.3. Let $A$ be a $C^{*}$-algebra and $T: A \rightarrow A$ a Riesz operator. If $T$ is a homomorphism with $\alpha(T)=p$, then $T^{p}$ is a finite rank operator.

Proof. Let $T$ be a Riesz operator with $\alpha(T)=p$. So $T^{p}$ is a Riesz operator too. If $N=N\left(T^{p}\right)$ then $\widetilde{A}=A / N$ is a $C^{*}$-Algebra because $N$ is a closed ideal of $A$. Consider the factorization $T^{p}=\widetilde{T^{p}} \circ \pi$, where $\pi: A \rightarrow \widetilde{A}$ is the canonical mapping and $\widetilde{T^{p}}: \widetilde{A} \rightarrow A$ is defined by $\widetilde{T^{p}}(x+N)=T^{p} x$ for all $x \in A$. If $\left(T^{p}\right)_{N}: \widetilde{A} \rightarrow \widetilde{A}$ is the induced map, then $\left(T^{p}\right)_{N}$ is a one-to-one Riesz operator and it is also a homomorphism. Therefore, $\left(T^{p}\right)_{N}$ has a closed range [3, Theorem 5.4]. Hence, the identity $\left.I=\left[\left(T^{p}\right)_{N}\right]^{-1} \circ\left[\left(T^{p}\right)_{N}\right)\right]$ is a Riesz operator. Thus, $\widetilde{A}$ is finite dimensional and consequently $T^{p}$ is a finite rank operator.

Corollary 3.4. Let $A$ be a $C^{*}$-algebra and $T: A \rightarrow A$ a Riesz operator. If $T$ is a homomorphism with $\alpha(T)=p$ and $N=N\left(T^{p}\right)$, then the induced operators $\left(T^{r}\right)_{N}$ are finite rank operators for $r=1,2, \ldots$

Proof. Since $\left(T^{r}\right)_{N}=\left(T_{N}\right)^{r}$ and the collection of finite rank operator is an ideal, it suffices to prove the statement for $r=1$. If $N=N\left(T^{p}\right)$ then $A / N$ is a $C^{*}$-algebra and $T_{N}$ is a well-defined Riesz operator [4, Theorem 3.23]. Note that $T_{N}$ is a monomorphism. Thus, our proof is complete using Proposition 3.2.

ACKNOWLEDGMENT. The authors gratefully acknowledge a grant from the NRF. Further, the authors wish to thank the referee for some helpful remarks.

\section{REFERENCES}

1. B. Aupetit, A primer on spectral theory (Springer, Berlin, Germany, 1991).

2. S. R. Caradus, W. E. Pfaffenberger and B. Yood, Calkin algebra and algebras of operators on Banach spaces, Lecture Notes in Pure and Applied Mathematics, vol. 9 (Marcel Dekker, New York, NY, 1974).

3. S. B. Cleveland, Homomorphism of non-commutative*-algebras, Pacific J. Math. 13 (1963), 1097-1109.

4. H. R. Dowson, Spectral theory of linear operators (Academic Press, London, 1978).

5. J. E. Gale, T. J. Ransford and M. C. White, Weakly compact homomorphisms, Proc. Amer. Math. Soc. 311(2) (1992), 815-824.

6. F. Ghahramani, Compact homomorphisms of C*-algebras, Proc. Amer. Math. Soc. 103 (1988), 458-461. 761-762.

7. M. Mathieu, Weakly compact homomorphisms, Proc. Amer. Math. Soc. 107 (1989), NJ, 1980). 